\title{
Application of Physics in Medicine
}

\author{
Syed Farooq Akber, Ph.D. \\ Houston, Texas
}

\begin{abstract}
The application of physics principles to medical practice has made an outstanding contribution in the diagnosis and treatment of cancer. Further amalgamation of research, application, cooperation and understanding of physics and medicine will enhance new perspectives on the benefits of ionizing and nonionizing radiations for medical diagnosis and therapy. In this paper, a general survey has been made of physics applications in medicine.
\end{abstract}

Key words: Medical imaging, NMR, MRS, radioisotope.

The application of physics principles to medical practice has made an astounding contribution in the prognosis and treatment of cancer. The discovery of X-rays by Roentgen ${ }^{1}$ in 1895 opened a new vista of medical imaging and was awarded the Nobel prize in physics in 1901. The discovery of artificial radioactivity by Joliot and Irene Curie ${ }^{2}$ in 1934 led John Lawrence two years later to inject a radioisotope (Phosphorus-32) into a patient to treat leukemia. In the $1950 \mathrm{~s}$, nuclear physicists Rosalyn Yalow and Solomon Berson ${ }^{3}$ developed the radioimmunoassay method of insulin, based on the principle of competitive binding by antibodies of natural and labeled hormones. This process is used by physiologists to visualize human organ functions. Rosalyn Yalow ${ }^{4}$ in 1977 received the Nobel Prize in medicine.

Physicists Cormack ${ }^{5}$ and Hounsfield ${ }^{6}$ earned the Nobel prize in medicine in 1979 for their independent and unique contributions to the invention of x-ray computed tomography (XCT). Walter Gilbert,' a theoretical physicist who did his doctorate under

From the Department of Radiology University of Texas Medical Center Houston, TX

Reprint Requests: Syed Farooq Akber

Department of Radiology

University of Texas Medical Center

6431 Fannin, 2.130 MSMB

Houston, $T X 77030$
Nobel laureate Professor Abdus Salam, developed an elegant technique for deciphering the genetic code, for which he was awarded the Nobel Prize in chemistry in 1980.

Thus evolved a new frontier of medical science known as "genetic engineering". No one would have thought that the concept of nuclear angular momentum introduced by Wolfgang Pauli, would be utilized by physicists 60 years later in the $1980 \mathrm{~s}$, for the visualization of anatomical and physiological functions of the human body. Physicists, on the other hand, using high energy accelerators for nuclear physics experiments, saw immediate application of such a method in the treatment of cancer. They modified these accelerators for clinical purposes providing several million electron volts $(\mathrm{MeV})$ of energy to treat cancer by ionizing radiation at any location in the body.

The widespread use of computers and digital imaging techniques in the 1970 s ushered in a revolution that continues to influence imaging modalities today. With these advances came both nuclear and x-ray imagers capable of producing true cross-section images of any surfaces.

Other areas of medicine in which physicists have made their contributions are ultrasound diagnosis, hyperthermia, audiology, opthalmology, endocrinology, physiology, physical therapy and general medical instrumentation. Laser scapels figure prominently as powerful tools in many surgical procedures. 


\section{Roentgenography}

A radiograph is a pictorial representation of human anatomy of $\mathrm{x}$-ray absorption by a three dimensional body onto a two dimensional film. Of all the imaging modalities to date, roentgenography features the best spatial resolution for high contrast objects. Even so, the vivid contrast is lost in the imaging of chest, breast and extremities for soft tissue characteristics, due to low differential attenuation of $\mathrm{x}$-ray photons. Contrast resolution also is limited by the scatter inherent in the attenuation process, and by the film-observer system dynamic range.

Interaction of matter with energy involves at least ten different processes. The amount of attenuation depends on the energy of the photons and the characteristics of the substances, such as atomic number, density and electrons per gram. X-ray photon energy and atomic number determine the relative percentage of photoelectric and Compton reactions. Density affects attenuation greatly, as evidenced in radiographic image contrast, due to tissue density variation.

\section{Dual energy subtraction roentgenography}

Tissue characteristic can be enahnced by the use of two photon energies. ${ }^{8}$ It provides spatial information as well as $\mathrm{x}$-ray intensity. The use of two photon energies provide us with two images simultaneously and can be resolved into water (soft tissue) and bone (mineral) components pixel by pixel. The intensity values are proportional to the overlying thickness of tissues and bone. A soft tissue image (that is, a bone canceled image) can be generated by computer by adding a small fraction of the bone image to the soft tissue image to patch up the voids left by the removal of bone. A single image can then be generated by adding the original low and high energy images together.

The advantage of the computer manipulated technique is a particular asset in chest roentgenography. It provides an image free from the disturbing influence of bone. For example, a nodule under the rib can be vividly seen on the soft tissue image, without the bone. This technique is also used to visualize the breast, the measurement of bone mineral content (for detecting and monitoring of osteoporosis), and the assessment of metabolic calcification of the lungs in kidney disease.

\section{X-Ray computed tomography (XCT)}

"Tomograph" is derived from the Greek "tomos", meaning "to slice", and "graph" meaning "picture". Thus a tomograph is a pictorial representation of a slice taken from a body. The $\mathrm{x}$-ray beam in computed tomography (CT) is collimated into a thin (1.5 to $10 \mathrm{~mm}$ ) fan that lies in a plane perpendicular to the long axis of the body. A circular array of detectors enclose the patient. The

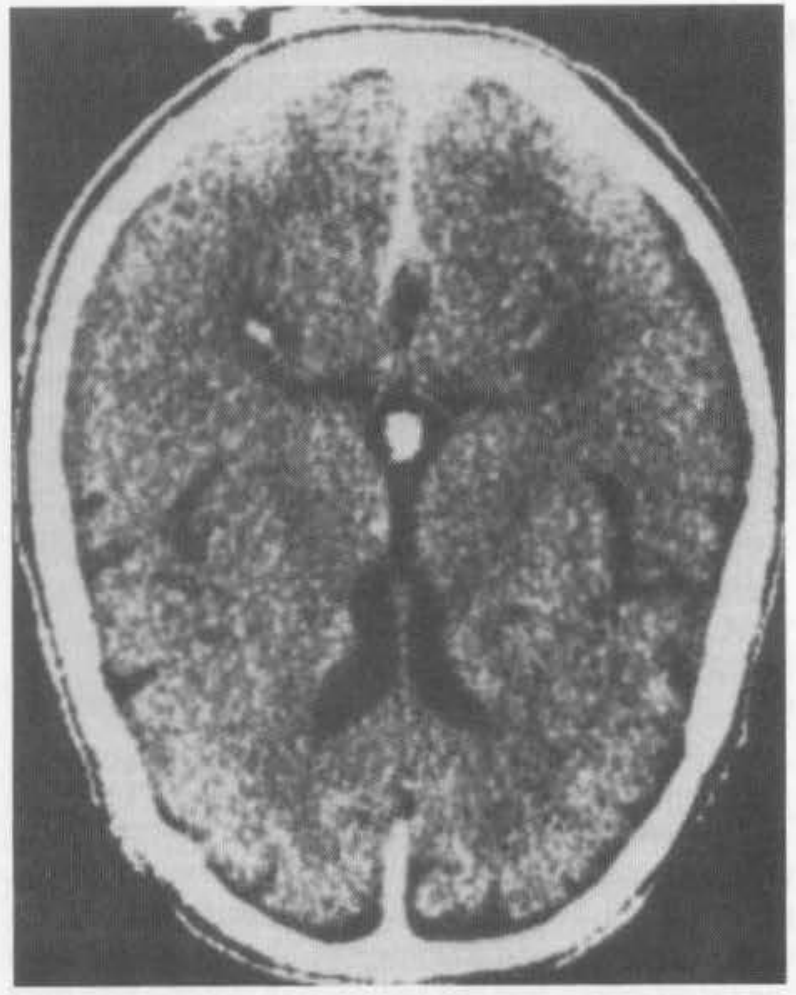

Figure 1. X-ray computed tomography image of the normal human brain, providing visual evidence of size, shape, position and symmetry of the fluid filled ventricles.

$\mathrm{x}$-ray tube revolves around the patient. The rotation of the $\mathrm{x}$-ray fan beam produces a charge signal proportional to the absorbed $\mathrm{x}$-ray energy in the detectors. The depth information is obtained by moving the $\mathrm{x}$-ray source about a fixed array of detectors and obtaining projections of the same section from $360^{\circ}$. Collection of data takes 1 to 10 seconds. Projection data is digitized, corrected for scatter, focal spot size and detector characteristics, and is reconstructed as a two dimensional map of the local attenuation coefficients, commonly known as CT numbers.

XCT is basically a diagnostic tool. It provides three dimensional information on the shape, dimensions and positions of anatomical structures. It offers scant value to physiologists in that it lacks the fourth dimension, temporal resolution.

$\mathrm{XCT}$ is indispensable in clinical practice. It is extremely useful in the diagnosis of central nervous system disease as the thickness of the human skull makes radiographic examination of the brain very difficult. XCT hurdles this barrier by providing visual evidence of size, shape, position and symmetry of the fluid-filled ventricles and cisterns critical to detection of hydrocephalus, brain atrophy, tumors, cysts, abscesses and edema (Figure 1). XCT images can be enhanced through use of contrast substances such as iodine to reveal hyperfusion or hypoperfusion, usully indicative of stroke or trauma. 


\section{Radionuclide imaging}

Nuclear medicine is the scientific and clinical discipline in which radiotracers are distributed in vivo when administered either orally or intravenously. The radiotracers for imaging purposes must satisfy the following criteria:

1. higher organ specificity

2. appropriate physical half-life that is compatible with the physiological half-life of the phenomenon under sutdy

3. highly abundant gammas of energies greater than $20 \mathrm{keV}$ for adequate tissue penetration and less than $500 \mathrm{keV}$ for higher sensitivity, better collimation, and minimal septal penetration

4. no particulate radiation

5. monoenergetic

6. should be nontoxic and taken up differently by normal and abnormal tissues.

In clinical nuclear medicine, radionuclides such as Technetium $99 \mathrm{~m}$, Iodine 123 , etc., are labeled to a chemical compound, which when administered to a patient, will localize within a specific organ. A chemical compound has specific binding sites within the target organ and the radioactive isotope acts only as an indicator of the physiological path of the drug. However, nuclear medicine technique does not depict structural anatomy as does ultrasound or XCT, or conventional radiographs. It is an established noninvasive technique available to investigate organ physiology, though NMR has shown its capability to investigate organ physiology and anatomy without ionizing radiation. ${ }^{9-11}$

Despite the many advances that have been made, the introduction of new radiopharmeceuticals has not been encouraging. The availability of new tracers results in increased application of radionuclide imaging. The introduction of Iodine 123 in recent years has provided new incentive to label biogenic amines, antibodies and free fatty acids. ${ }^{12}$ While biogenic amines and free fatty acids have already found their way into clinical diagnostic inventories, antibodies for in vivo detection of tumors are now used in clinical trials.

The most promising of all biogenic amines is $\mathrm{N}$-Isopropyl-I-123-p-Iodoamphetamine (I-123 IMP). I-123 IMP binds to nonspecific sites after intravenous administration in the brain in accordance with regional cerebral blood flow. It has also been used to study cerebrovascular disorders and metabolic activities of the brain using SPECT, and has been very successful in the detection of Alzhemier's disease.

I-123 IMP is also retained in the lung. ${ }^{13-15}$ Akber $^{16}$ has modified the Chinard-Crone's invasive indicator dilution technique and developed a totally noninvasive in vivo technique using convolution and deconvolution analysis using nuclear medicine methodology. Akber ${ }^{17-18}$ provided further evidence of endothelial amine receptors by demonstrating the active competition between I-123 IMP and propranolol and I-123 IMP and ketamine. This was the first noninvasive in vivo radioimmunoassay technique, demonstrated by Akber, ${ }^{19}$ between two biogenic amines of different chemical structure with similar binding sites in the lung. This technique has opened a new vista to assess pulmonary endothelial receptors in the lungs in vivo. It is well known that pulmonary endothelial amine receptors are the primary site of damage in a number of disease states such as oxygen toxicity, adult respiratory distress syndrome and a variety of toxin and drug induced lung injuries. Assessment of pulmonary endothelial receptor sites in normal and pathological condition is an important step in understanding of receptor related diseases. With currently available radiotracers and detectors (Anger scintillation camera, single photon computed tomgraphy, and positron computed tomography) it is possible to scan, either in static mode or dynamic mode, the brain, the lungs, the liver, bones, the kidneys, the spleen and tumors. Akber ${ }^{20}$ has discussed the various aspects of radionuclide imaging and therefore, we will restrict our discussion to positron emission tomography (PET).

A number of radionuclides decay through the emission of positrons. A positron combines with an electron, which in turn are annihilatd into two photons of $511 \mathrm{keV}$ each, at a $180^{\circ}$ angle from each other. These photons can be detected by two scintillation detectors connected in a coincidence circuit. A field of view is established that does not vary with depth in PET. The attenuation can be computed by measuring the thickness of the object traversed (assuming a uniform absorbent), or by measuring its absorption through placement of a position-emitting source outside the integral line.

PET yields sectional images of the distribution of positron-emitting radionuclides in an organ of the patient in an analogous manner as XCT. The only difference is that in PET the $\mathrm{x}$-ray source is replaced by the body of a patient, injected with a radiopharmaceutical,which acts as a source, surrounded by a ring of detectors.

PET provides an analytical and noninvasive technique for studying brain, heart, lungs, or pancreas. Physiological and biochemical functions can be visualized by the use of physiologically active compounds labeled with positron emitting radionuclides such as carbon-11, nitrogen-13, oxygen-15, or fluorine-19. The assessment of regional blood flow, oxygen consumption, variety of metabolic activities, transport and hemodynamics, and neuroreceptor mapping have enabled us to examine ischemia and several degenerative disorders such as Huntington's disease, Alzhemier's disease, schizophrenia and Parkinson disease. 


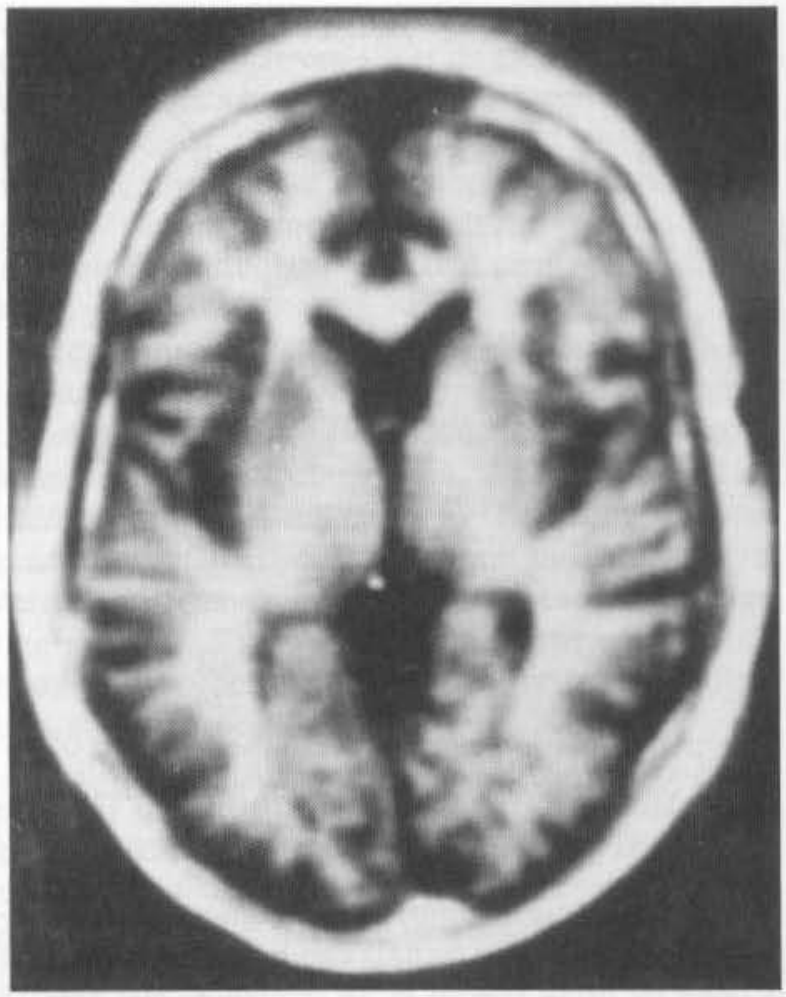

Figure 2. Precession of nuclear angular momentum (L) of proton about the direction of static magnetic field $\left(\mathrm{H}_{\mathrm{O}}\right)$.

\section{Nuclear magnetic resonance imaging (NMR)}

Nuclear magnetic resonance (NMR) is a nuclear phenomena. All nuclei with odd numbers of protons or neutrons, or both protons and neutrons of an element such as hydrogen in water, have nuclear spin $(I=1 / 2)$. When spinning nuclei are subjected to a strong magnetic field, the nuclei will align themselves with the magnetic field and precess along the external magnetic field with a frequency proportional to the magnetic field strength (Figure 2). This precession is called Larmor frequency. The application of a radiofrequency excitation energy to the precessing nuclei will flip the magnetic moment at an angle $\boldsymbol{Q}$ When the radiofrequency of precession is equal to Larmor frequency, then resonance will occur, hence the phenomena is called nuclear magnetic resonance. When the radiofrequency is stopped, the nuclei will return to their original positions. In so doing, the nuclei will emit radiofrequency waves of the energy absorbed. These energy waves can be externally detected by the radiofrequency coils enclosing the patient, generating the NMR signal that is reconstructed to form an image. ${ }^{21}$

When the radiofrequency is turned off, two processes occurs at the same time, which are commonly known as relaxation processes.

1. The equilibrium magnetization in the direction of magnetic field $\mathrm{Ho}$ is recovered as the stimulated spins emit their energy to their surroundings, i.e., to the lattice. The time constant for this process is called spin lattice relaxation time (T1).

2. The phase coherence is lost through spin-spin exchange processes. In this, two nuclear spins, lower and upper state, flip simultaneously. The phase incoherence is contributed by the inhomogeneity of the magnetic field $\mathrm{Ho}$, which leads to differing Larmor frequencies of individual spins. The time constant for this process is known as spin-spin relaxation time (T2).

Contrast in NMR images is based on relaxation time dissimilarities between normal and malignant tissues. However, no positive correlation has been provided by the NMR investigators as to why the NMR relaxation times are different in normal and pathological states. Akber ${ }^{22-27}$ has proposed an hypothesis to explain this enigma: the dissimilarities in relaxation time is due to the concentration of paramagnetic molecular oxygen dissolved in cell water. Under the purview of this hypothesis, Akber ${ }^{28-29}$ has also recently shown that the variation in relaxation times from organ to organ is due to the consumption of oxygen.

One of the advantages of NMR imaging is that it obtains high contrast images compared to XCT. In NMR imaging, brain tissue divides into two classes: gray and white matter. Gray matter appears as a collection of nerve cell bodies and white matter consists of nerve fibers. Hydrogen in gray matter is nearly all water, whereas in white matter a significant amount is contained in lipids. A vivid contrast can be seen between gray and white matter due to signal intensity differences (Figure 3 ) which makes it possible to define internal structures of the brain with a clarity not seen by XCT. The reason XCT does not distinguish between gray and white matter so vividly is because the attenuation difference of $\mathrm{x}$-ray photons is $0.5 \%$, one of the factors inhibiting image enhancement in XCT.

NMR imaging has been described as a quantum leap in medical imaging. By use of gradient coils in NMR imagers, one can generate images in sagittal, coronal and transverse planes. The advantage of NMR imaging can clearly be seen in the central nervous system. It excels in this domain over all other imaging modalities. For other anatomic regions such as the cardiovascular system, angiography, helim, mediastinum, kidneys and orbits (eye), NMR imaging has shown its potential to surpass other imaging techniques. Among current advances in NMR imaging is the development of the surface coil. The signalto-noise ratio can be enhanced by the use of the surface coil when placed close to the region of interest such as the eye, ear, spine, knee, or breast.

NMR imagers are also available with spectroscopy capability. This enables physicists and clinicians to 


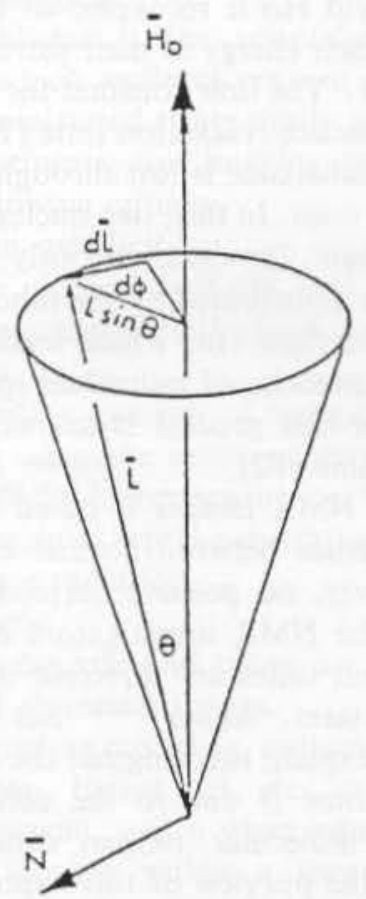

Figure 3. NMR image of the normal human brain, showing vivid distinction between gray and white matter.

noninvasively monitor the biochemical processes of human organs in normal and pathological states.

Magnetic resonance spectroscopy (MRS) has been developed over the past 30 years as an analytical tool in chemistry. The sole objective of MRS is to extract information about the molecular structure, configuration, and dynamics of chemical compounds. MRS can also be applied noninvasively to study metabolism and physiology in living tissues and organisms. The nuclei that are of biological importance and which have magnetic moments are P-31, $\mathrm{Na}-23, \mathrm{C}-13$, and $\mathrm{N}-14$.

The biochemical process of phosphorus in living beings provides information about the metabolites of phosphates such as phosphocreatine (PCr), adenosinetriphosphate (ATP), and inorganic phosphate (Pi) using P-31 MR spectroscopy. Assessment of tumors using MRS provides information regarding tumor perfusion. Okunieff et $\mathrm{al}^{30}$ assessed the P-31 spectra of murine tumors in vivo in rat at $10 \%, 21 \%$, and $100 \%$ of inspired oxygen. Figure 4 shows a linear relationship between percent of inspired oxygen and $\mathrm{PCr} / \mathrm{Pi}$ ratio. Gyulai et al, ${ }^{31}$ from their P-31 studies on graded hypoxia and hyperoxia in vivo in the gerbil brain, showed a decease in $\mathrm{PCr} / \mathrm{Pi}$ ratio at differing percentages of inspired oxygen. Similarly, P-31 spectra before and after successful radiation treatment of the tumor reveals normal spectra, clearly favoring the concept of normal perfusion after radiation treatment.

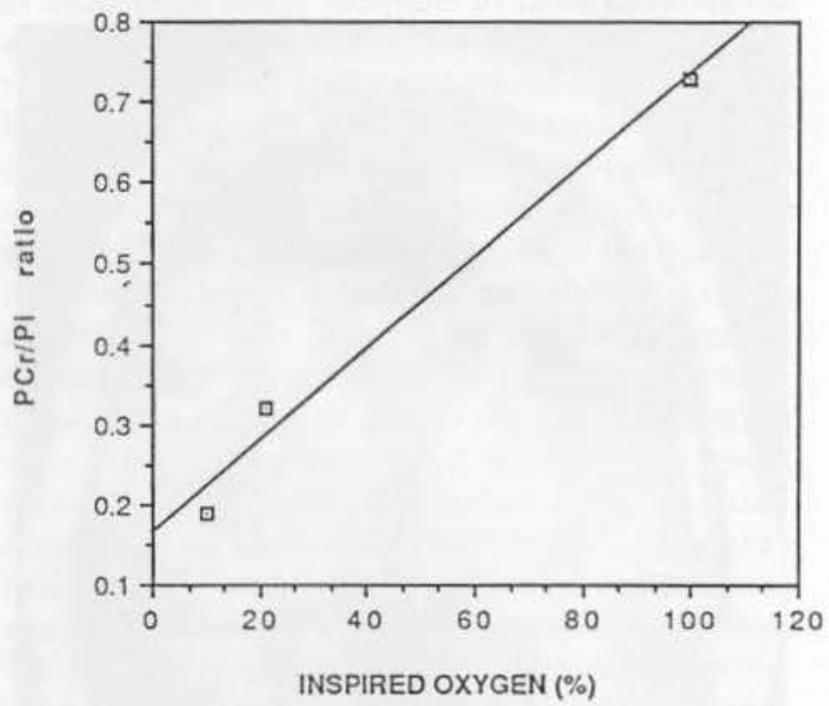

Figure 4. Relationship between the percent of inspired oxygen and $\mathrm{PCr} / \mathrm{Pi}$ ratio in murine tumors.

H-1 NMR spectroscopy can be observed if the intense water and lipid signals can be suppressed. This is done by using special pulse sequence. $\mathrm{H}-1$ spectra measure various metabolities such as lactate, $\mathrm{N}$-acetylaspartate and amino acids. The $\mathrm{C}-13$ spectra on the other hand, characterize high concentration of lipids and gylcogen. The use of C-13 labeled subtrates also allows the monitoring of metabolism by the C-13 NMR spectroscopy.

MRS is not, however, free of constraints. The two most important limiting factors are sensitivity and resolution. To enhacne both spatial resolution and sensitivity, higher magnetic field are necessary. But, given the constraint that the magnetic field strength ranges from 0.3 to $1.5 \mathrm{~T}$, the decrease in signal intensity can be compensated by the use of a surface coil, signal averaging and large volume of interest.

New applications of NMR imaging are now seen in geology, agronomy, botany and material science. NMR imaging is a very suitable technique for studying plant root systems within the soil. They can be observed for transportation of water, sunlight influence, water stress and disease. NMR also may provide a useful index for assessing the influence of carbon dioxide, pollutants, chemicals and herbicides, as well as optimizing growth conditions in seed beds. This technique would be of immense value to Hakims, who use herbs and plant roots in their medication. This will provide them an opportunity to evaluate the potency of herbs and roots and their moisture content and relation to potency of the roots and herbs.

\section{Ultrasound}

In medical sonography, frequencies between 1 $\mathrm{MHz}$ and $20 \mathrm{MHz}$ are used. Such high frequencies 
are generated by piezoelectric crystals made either of quartz or synthetic ceramic. The piezoelectric crystals are sandwiched between silver or gold electrodes and are enclosed in a box called a transducer. If voltage is applied across the crystal face and then quickly removed, the crystal will vibrate. This vibrating crystal, coupled with the body, gives rise to an ultrasonic longitudinal wave that propagates into the body.

The transducer in sonography acts both as transmitter and as receiver. A short ring down time is therefore essential for the production of high quality images. In a pulse echo system, the transducer is excited once every millisecond. The time between generation of the first and second pulse is used for detection of returning echoes from the body reflecting surfaces. The sound beam is attenuated in the body by the processes of diffusion, reflection, refraction and absorption.

In diagnostic applications of sonography, use is made of reflected ultrasound waves from interfaces between different tissues in the patient. The part of the incident energy reflected from an interface depends on the difference in acoustic impedance of the media on the other side of the interface. The acoustic impedance of a medium is given by the formula:

$$
z=\rho C
$$

where $\mathcal{f}$ is the density of the medium and $C$ is the velocity of ultrasound in the medium. The greater the discrepencies in acoustic impedance of the two media, the larger part of the incidence beam is determined by the angle of incidence of the beam at the interface. The higher the angle of incidence, i.e., close to $90^{\circ}$, the lesser the amount of reflected beam. Below a certain critical angle, the entire beam is reflected. The reflected beam can be written as follows:

$$
\mathrm{R}=[(\mathrm{Z} 2-\mathrm{Z} 1) /(\mathrm{Z} 1+\mathrm{Z} 2)]^{2}
$$

where $\mathrm{Zi}$ and $\mathrm{Z2}$ are the acoustic impedance of medium 1 and 2. The part of the incident energy transmitted through an interface is:

$$
\mathrm{T}=4 \mathrm{Z} 1 \mathrm{Z} 2 /(\mathrm{Z} 1+\mathrm{Z} 2)^{2}
$$

However, $\mathrm{R}+\mathrm{T}=1$.

At the muscle-liver interface only $1 \%$ of the beam is reflected while $99 \%$ transmitted. Even though $1 \%$ of the total beam reflected seems small, it nevertheless depicts the profile of the liver. In the case of chest walls and the lungs, the millions of air tissue interfaces within the lungs provide a greater degree of impedance mismatch at the interface. This results in strong reflection of the ultrasound beam at the boundary, which prevents the clinical applications of ultrasound imaging of the lung. This is likewise the case with soft tissues and bone.

To avoid high reflection of an ultrasound beam at the air-tissue interface, water paths, cream or gels are used to eliminate the air pockets which block good acoustic coupling between the transducer and the patient's skin.

The velocity of the sound beam changes as it traverses from one medium to another. However, the frequency remains constant, though the wavelength changes. If the ultrasound beam strikes a second medium at an angle, the beam is refracted, or bent. The degree of refraction depends on the angle of incidence and difference in the velocity of sound in the two media. Refraction is a major cause of artifacts in images obtained from ultrasound.

Frequencies can help differentiate between tissues. Use of higher frequencies results in better resolution. However, the use of higher frequencies results in poor beam penetration of the organ. Attenuation is approximately linearly proportional to the frequency.

With the many advances made in ultrasonography instrumentation, the state-of-the-art instrument is the Duplex ultrasound. It is a high resolution, realtime, B-mode imaging system with pulsed Doppler color flow imaging. Linear array transducers or mechanical sector transducers are used to generate color flow images by computing the amplitude, phase and frequency of ultrasound signals relected from the tissue. Stationary or slow moving tissue signals are not eliminated from the image formation in the realtime. The reflected ultrasound signals from the tissue are assigned a gray scale which is proportional to the echo signal amplitude. The phase of the echo, on the other hand, provides information regarding the presence and direction of motion. Color assignments are made based on phase changes. For example, red represents flow away from the transducer while blue represents flow towards the transducer. Different shades of red and blue represent arterial and venous flow. The shades of either blue or red depend on the velocity of the blood flow. Maximum Doppler shift is represented by white, representing maximum velocity. The image obtained by this system represents the hemodynamic characteristics of flow within the vessel. Gomez et $\mathrm{al}^{32}$ has discussed the technical background and clinical applications of color flow imaging.

There are clinical applications of ultrasound scanning in obstetrics, gynecology, abdominal and pelvic pathology, cardiology, opthalmology and oncology. Although the image quality of ultrasound is not very good, it nevertheless, offers a valuable diagnostic tool for imaging soft tissues without the use of ionizing radiation. Visualization of gall stones by this technique has been performed with $100 \%$ assurance. Study of the growth of the fetus is conducted routinely in most departments of obstetrics. 


\section{Radiotherapy}

The established clinical practice of radiation therapy uses non-invasive, external ionizing radiation beams (x-rays, gamma rays from cobalt 60 , and electron beams) for the treatment of tumors and cancer in morbid persons. The purpose of radiotherapy is to achieve a concentrated radiation dose to the tumor and yet to avoid damage to healthy tissue. While making treatment plans physicists have to be very prudent to ensure that radiation sensitive organs such as the eyes, spinal cord, kidneys and heart, receive as little radiation as possible. If the patient receives over 4500 rads on his spinal cord because of improper treatment planning, the patient may be paralyzed.

Neutron beams and negative pions therapy are in the offing. ${ }^{33}$ Advances in radiation therapy are likely to occur in areas such as the combination of chemotherapy-radiotherapy, hyperthermiaradiotherapy and modification of radiation dose fractionation schemes. Since diagnosis of malignant diseases often is made at advanced stages, treatment of such diseases is a difficult proposition.

Another method for treating tumors using ionizing radiation internally is brachytherapy. Radium and radon seeds were used but are now being replaced by Co-60, Cs-137, Ir-192 and I-125. These radionuclides are encapsulated in needle and tubes. The sources in moulds or placques are located on superficial lesions inserted interstitially in the tissue of the lesion or nearby, or is placed within natural body cavities. In order to enhance the tumor dose, both external and internal treatment occasionally are carried out.

\section{Conclusion}

Physicists and medical physicists laid the cornerstone in the diagnosis and treatment of cancer in today's medical practice. Further coalescence of research, application, cooperation, and understanding will enhance new perspectives on benefits of ionizing and nonionizing radiations for medical diagnosis and therapy of cancer. The advances in medicine will continue to depend on the foresight of physicists and physicians who will amalgamate instrumentation, physics, chemistry, mathematics and physiology into medicine.

\section{References}

1. Roentgen WC. Ubereine neue Art von Strahlen (vorlaufig Mitteilung), sitzungs-Berichte der Physikalish medicinischen Gesellschaftzu Wurzburg .1895; 9:132.

2. Curie I, Joliot F. Nature. 1934; 133:201.

3. Berson SA, Yalow RS, et al. Insulin I-131 metabolism in human subjects: Demonstration of insulin treated subjects. J Clin Invest. 1956; 35:170-190.

4. Yalow RS. Radioimmunoassay: A probe for the fine structure of biologic system. Science.
1978;35:1236-1245.

5. Cormack AM. Representation of a function by its line integrals with some radiological applications. J Appl Phys. 1963; 34:2722.

6. Hounsfield GN. Computerized transverse axial scanning (tomography). Br J Radiol. 1973; 46:1016.

7. Jacobson B. Acta Radiol. 1953;39:436.

8. Gilbert W Science. 1981;214:1305.

9. Akber SF. Nuclear medical resonance medical imaging. JPMA. 1984;34:64-71.

10. Akber SF. The physics of NMR tomography. Nuklearmedizin. 1986;25:33-37.

11. Akber SF. Clinical ramifications of NMR imaging. Compr Ther. 1987;13:41-45.

12. Akber SF. Clinical ramifications of iodine. Compr Ther. 1987;13:19-25.

13. Akber SF. Lung as a metabolic organ. Editorial. JPMA. 1986;36:132-136.

14. Touyo J, Akber SF, J Rahimian J, Bennett LR. Metabolic lung scanning with N-Isopropyl-I-123p-Iodoamphetamine. In: Nuclear Medicine and Biology, Proceedings of the Third World Congress of Nuclear Medicine and Biology, Raynaud C, ed. Paris: Pergamon Press III, 1982, pp. 2554-2557.

15. Rahimian J, Glass EC, Touya JJ, Akber SF, Graham LS, Bennet LR. Measurement of metabolic extraction of tracers in the lung using a multiple indicator dilution technique. J Nucl Med. 1984;25:31-37.

16. Akber SF. On the theory of the first pass pulmonary extraction using indicator dilution technique. Nuklearmedizin. 1987;26:21-27.

17. Akber SF. Ketamine influence on the first pass pulmonary extraction of N-Isopropyl-I-123-plodoamphetamine using indicator dilution technique. Proceedings of the 14th International Conference on Medical and Biological Engineering and 7 th International Conference on Medical Physics. Espoo, Finland, August 11-16, 1985. Med Biol Engin Compt 23: (Suppl. Part 2):1377-1378, 1985 .

18. Akber SF. Demonstration and quantitation of pharmacological inhibtion of pulmonary uptake of N-Isopropyl-I-123-p-Iodoamphetamine by propranolol. Nuklearmedizin. 1989;28:100-104.

19. Akber SF. A noninvasive assessment of pharmacological interaction of amines in the lung. Nucl Med Commun. 1987;8:889-893.

20. Akber SF. Radionuclide imaging. JPMA. 1986;36:151-153.

21. Akber SF. From NMR signal to NMR tomography. Europ J Radiol. (In press)

22. Akber SF. Comment on "A review of $\mathrm{H}-1$ nuclear magnetic resonance relaxation in pathology: Are T1 and T2 diagnostic?" Med Phys. 1987;14:1090-1091. 
23. Akber SF. The quagmire of relaxation times. Med Hypotheses. 1988;26:187-191.

24. Akber SF. The conundrum of relaxation times. Nuklearmedizin. 1988;27:43-45.

25. Akber SF. Influence of anesthetics on relaxation times. Anesthesiology. 1988;69:290-291.

26. Akber SF. Correlation between oxygen tension and spin lattice relaxation rate in tumors. Europ J Radiol. 1989;9:56-59.

27. Akber SF, A correlation between spin lattice relaxation time and tumor growth. Europ J Radio. 1989;9.

28. Akber SF. Physiologic basis of spin lattice relaxation time in normal rat tissues. Proceedings 11th International Conf of IEEE in Medicine and Biology. Nov., 1989, Seattle, Washington.

29. Akber SF. The enigma of relaxation times in normal and malignant human tissues. Studia Biophysica 1990. (In press)
30. Okunieff P, Ramsay J, Tokuhiro T, Hutzig BM, Rummery E, Neuringer LJ, Snit H. Estimation of tumor oxygenation and metabolic rate using P-31 MRS: Correlation of Longitudinal relaxation with tumor growth rate and DNA synthesis. Int J Radiat Oncol Biol Phys. 1988;14:1185-1195.

31. Gyulai L, Chang B, Ligeti L, McDonald G, Cone J. Correlated in vivo P-31 NMR and NADH fluorometric studies on gerbil brain in graded hypoxia and hyperoxia. Am J Physiol. 1988;254 (Cell Physiol 23): C699-C708.

32. Gomez CR, Fischer AQ, Gomez SM. Color flow imaging in neurosonology: Technical background and clinical applications. JIMA. 1988;20:141-145.

33. Akber SF. Neutron therapy: Of what clinical relevance? Compr Ther. 1988;14:3-7. 